\title{
AN ADAPTIVE MULTI-LEVEL METHOD FOR CONVECTION DIFFUSION PROBLEMS *
}

\author{
Martine Marion $^{1}$ And Adeline Mollard ${ }^{2}$
}

\begin{abstract}
In this article we introduce an adaptive multi-level method in space and time for convection diffusion problems. The scheme is based on a multi-level spatial splitting and the use of different timesteps. The temporal discretization relies on the characteristics method. We derive an a posteriori error estimate and design a corresponding adaptive algorithm. The efficiency of the multi-level method is illustrated by numerical experiments, in particular for a convection-dominated problem.
\end{abstract}

Mathematics Subject Classification. 65M15, 65M25, 65M70, 76R99.

Received: October 8, 1999. Revised: December 13, 1999.

\section{INTRODUCTION}

The aim of this article is to present some a posteriori error estimates and numerical results that show the interest of adaptive multi-level techniques for the approximation of (linear) convection diffusion problems.

The spatial discretization we consider is of Fourier type. Such an approximation allows to simply define a two-level spatial decomposition. But, we believe that the techniques extend to other types of discretization; see Marion-Xu [13] for a first step in that direction.

The time discretization relies on the characteristics method. It is well known that the approximation of convection (dominated) diffusion method is a difficult question due to the nearly hyperbolic nature of the equations. In the characteristics methods, the governing equations are written in terms of Lagrangian coordinates as defined by the particle trajectories (or characteristics). Then the Lagrangian total derivative is approximated thanks to some divided difference operator. These methods have largely proven their efficiency (see for example $[1,2,6,15,16]$

Let $T>0$. We consider a (not necessarily uniform) subdivision of $(0, T)$ : $0=T_{0}<T_{1}<\ldots<T_{Q-1}<$ $T_{Q}=T$. On the time interval $J_{q}=\left(T_{q-1}, T_{q}\right)$, the approximate solution lies in the space $S_{M_{q}}$ of trigonometric polynomials of degree $\leq M_{q}$ in each variable. The multi-level strategy consists in introducing a cut-off value $1 \leq m_{q} \leq M_{q}$ and looking for an approximate solution of the form

$$
V+W \text {, with } V \in S_{m_{q}} \text { and } W \in\left(I-P_{m_{q}}\right) S_{M_{q}} .
$$

\footnotetext{
Keywords and phrases. Multi-level methods, convection-diffusion equations, characteristics method, a posteriori error estimates, adaptive algorithms.

* Dedicated to Roger Temam for his 60th birthday

${ }^{1}$ UMR CNRS 5585, Département Mathématique-Informatique, École Centrale de Lyon, BP 163, 69131 Écully Cedex, France.

${ }^{2}$ UMR CNRS 5640, Département Génie Mathématique, INSA, Complexe de Rangueil, 31077 Toulouse Cedex 4, France.
} 
Here, the high modes component $W\left(=W_{q}\right)$ is integrated with the time-steps $K_{q}=T_{q}-T_{q-1}$ whereas the low modes component $V$ is integrated with a smaller time-step. This means that the above subdivision of $(0, T)$ is refined into $0=t_{0}<t_{1}<\ldots<t_{N-1}<t_{N}=T$ and the component $V\left(=V_{n}\right)$ is re-evaluated at the intermediate time-steps $t_{n}$ with $I_{n}=\left(t_{n-1}, t_{n}\right) \subset J_{q}$. Consequently the scheme involves two families of discretized characteristics curves associated respectively to the time-steps $k_{n}=t_{n}-t_{n-1}$ and $K_{q}$. The algorithm is described in Section 2.

The motivation for such a strategy stems from Foias-Manley-Temam [9]. For 2D Navier-Stokes equations, these authors showed that the energy carried in the higher modes of the solution is much smaller than the one carried in the lower modes. Consequently, the contribution to the error of the higher modes should be small. Therefore, it seems natural to integrate them with a larger time-step and hope not to spoil the overall accuracy. Various works for parabolic problems confirmed this strategy $[3-5,7,11]$

The extension of such a strategy to convection dominated problems is not straightforward and this was the aim of Marion-Mollard [12] where a non adaptive multi-level method for convection diffusion problems was introduced. A priori error estimates and numerical tests showed that, for an appropriate choice of $m$ and $K$, the error is similar to the one of the classical (one-level) method with the fine discretization parameters $M$ and $k$.

Clearly, the multi-level method involves numerous parameters that have to be carefully chosen. Therefore, it is natural to ask for an adaptive algorithm to determine these parameters. This could allow an automatic and more efficient choice as well as time varying values. Also, in order to investigate the interest of the multi-level approach, the comparison of the numerical performances of the algorithm with the ones of a one-level adaptive algorithm is an interesting step.

In Section 3 we first derive some a posteriori error estimate. Our techniques are inspired by the works of Eriksson-Johnson [8] and Houston-Suli [10] for finite elements methods. They include a representation of the error involving a dual problem, the derivation of strong stability estimates and the use of Galerkin orthogonality. We derive a bound of the error in the norm of $L^{2}\left(0, T ; L^{2}(\Omega)\right)$. Of course we are also able to obtain a bound for the (simpler) one-level adaptive method.

Next in Section 4, we design the corresponding adaptive algorithms (both in the one-level and the multi-level cases). The algorithm finds all discretization parameters $M_{q}, m_{q}, K_{q}$ and $k_{n}$ so that the norm of the error is below some given tolerance $T O L$. The procedure is based on an appropriate splitting of the estimator and also of TOL.

Numerical tests for one-dimensional problems are presented in Section 5. The stability of the algorithm for convection dominated problems and the interest of adaptivity are evidenced. Concerning computing time, the multi-level procedure allows a gain up to $65 \%$ with respect to the one-level adaptive scheme.

This gain is in particular due to the use of FFTs of different orders in the multi-level procedure. Therefore, it should increase with the space dimension. In a subsequent work we intend to present numerical results in higher space dimension.

\section{ThE EQUATION AND ITS DISCRETIZATION}

Let $\Omega=] 0,2 \pi^{d}, d=2,3$, and $T>0$. We consider the following linear unsteady convection-diffusion problem involving a function $u$ from $\Omega \times[0, T]$ into $\mathbb{R}$

$$
\begin{gathered}
\left.\frac{\partial u}{\partial t}+a . \nabla u-\nu \Delta u=f, \text { in } \Omega \times\right] 0, T[, \\
u(x, 0)=u_{0}(x), x \in \Omega,
\end{gathered}
$$


Here $\nu>0$ is the viscosity, $f$ is the forcing term and $a$ is some divergence free vector field ${ }^{1}$.

It is well known that under the assumptions

$$
u_{0} \in L^{2}(\Omega), f \in L^{2}\left(0, T ; L^{2}(\Omega)\right), a \in L^{\infty}\left(0, T ; W_{p}^{1, \infty}(\Omega)^{d}\right) \text { with } \operatorname{div} a=0
$$

problem (2.1)-(2.3) possesses a unique solution $u \in L^{2}\left(0, T ; H_{p}^{1}(\Omega)\right) \cap \mathcal{C}\left([0, T] ; L^{2}(\Omega)\right)$.

We first describe a one-level code for the integration of (2.1). It is inspired from the work of Houston-Suli [10] for finite elements approximations.

The spatial discretization relies on the space $S_{M}$ of trigonometric polynomials of degree $\leq M$ in each variable. We denote by $P_{M}$ the $L^{2}(\Omega)$ projection onto $S_{M}$.

Let $0=t_{0}<t_{1}<t_{2}<\ldots<t_{N}=T$ be a subdivision (not necessarily uniform) of $(0, T)$ with the corresponding time intervals $I_{n}=\left(t_{n-1}, t_{n}\right)$ and the time-steps $k_{n}=t_{n}-t_{n-1}$. On each time interval $I_{n}$, the solution $u$ of problem (2.1) is approximated by $U_{n} \in S_{M_{n}}$.

The time discretization uses particle trajectories (or characteristics) associated with problem (2.1). Recall that the path of a particle located at $x \in \bar{\Omega}$ at time $s \in[0, T]$ is defined as the solution of the initial value problem

$$
\left\{\begin{array}{l}
\frac{\mathrm{d}}{\mathrm{d} t} X(x, s ; t)=a(X(x, s ; t), t) x \in \Omega, t \in[0, T] \backslash\{s\} \\
X(x, s ; s)=x
\end{array}\right.
$$

Then setting

$$
X_{n-1}=X\left(x, t_{n} ; t_{n-1}\right)
$$

the sequence $\left(U_{n}\right)_{n}$ is given by the following recursive formula

$$
U_{n}(x)-P_{M_{n}} U_{n-1}\left(X_{n-1}(x)\right)-k_{n} \nu \Delta U_{n}(x)=k_{n} P_{M_{n}} f\left(x, t_{n}\right)
$$

where

$$
U_{0}=P_{M_{0}} u_{0}
$$

Next we aim to introduce a multi-level procedure. The strategy consists in freezing the higher modes of the approximate solution during several iterations of the lower modes. As explained in the introduction, we expect that integrating the higher modes with a larger time-step will not spoil the accuracy of the method.

As above we use the decomposition of $(0, T)$ in $N$ sub-intervals $I_{n}=\left(t_{n-1}, t_{n}\right)$ with $k_{n}=t_{n}-t_{n-1}$. We also introduce another decomposition of $(0, T), 0=T_{0}<T_{1}<T_{2}<\ldots<T_{Q}=T$ with the corresponding time intervals $\left.J_{q}=\right] T_{q-1}, T_{q}$ [ and the time-steps $K_{q}=T_{q}-T_{q-1}$. Here, each $J_{q}$ is the union of successive $I_{n}$. It is convenient to introduce the largest $n$ such that $I_{n} \subset J_{q}$, that we denote by $n_{q}$. Hence, $T_{q}=t_{n_{q}}$.

On the time interval $J_{q}$, the approximate solution lies in $S_{M_{q}}$. We introduce a cut-off mode $m_{q}$ with $1 \leq m_{q} \leq M_{q}$ and write

$$
S_{M_{q}}=S_{m_{q}}+\left(I-P_{m_{q}}\right) S_{M_{q}}
$$

Correspondingly, the approximate solution splits into the sum of a low frequency term and a high frequency one. More precisely, on some time interval $I_{n} \subset J_{q}$, the approximate solution reads

$$
V_{n}+W_{q} \text {, with } V_{n} \in S_{m_{q}} \text { and } W_{q} \in\left(I-P_{m_{q}}\right) S_{M_{q}} .
$$

\footnotetext{
${ }^{1}$ The condition $\operatorname{div} a=0$ is not compulsory and is only introduced for simplicity.
} 
The component $V_{n}$ is advanced in time thanks to the (small) time-step $k_{n}$ while the component $W_{q}$ is advanced in time thanks to the (large) time-step $K_{q}$. Hence, we consider the two families of characteristics curves

$$
X_{n-1}=X\left(x, t_{n} ; t_{n-1}\right), n=1, \ldots, N, \text { and } Y_{q-1}=X\left(x, T_{q} ; T_{q-1}\right), q=1, \ldots, Q .
$$

With these notations the two-level algorithm reads

$$
\begin{aligned}
& V_{n}-P_{m_{q}}\left(V_{n-1}+W_{q-1}\right)\left(X_{n-1}\right)-k_{n} \nu \Delta V_{n}=k_{n} P_{m_{q}} f\left(t_{n}\right), \\
& n=1, \ldots, N, I_{n} \subset J_{q} \\
& W_{q}-\left(P_{M_{q}}-P_{m_{q}}\right)\left(V_{n_{q-1}}+W_{q-1}\right)\left(Y_{q-1}\right)-K_{q} \nu \Delta W_{q}=K_{q}\left(P_{M_{q}}-P_{m_{q}}\right) f\left(T_{q}\right), \\
& q=1, \ldots, Q,
\end{aligned}
$$

with

$$
V_{0}=P_{m_{0}} u_{0}, W_{0}=\left(P_{M_{0}}-P_{m_{0}}\right) u_{0} .
$$

Here, on the time interval $J_{q}$, one needs to solve the equation (2.8b) and successively the equation (2.8a) for the appropriate values of $n$. Note that this algorithm is an adaptive version of the one introduced in MarionMollard [12].

\section{A POSTERIORI ERROR ANALYSIS}

The aim of this section is to derive an a posteriori error estimate for the algorithm (2.8). This estimate depends on the data, the approximate solution and the discretization parameters.

At time $t \in I_{n} \subset J_{q}$, the error reads

$$
e(t)=u(t)-\left(V_{n}+W_{q}\right) .
$$

It is convenient to introduce the functions $m, M, k, K$ defined on $(0, T)$ by

$$
m(t)=m_{q}, M(t)=M_{q}, k(t)=k_{n}, K(t)=K_{q} \text { for } t \in I_{n} \subset J_{q} .
$$

Also we denote by |.|, (.,.) the norm and product scalar of $L^{2}(\Omega)$ and by $|\cdot|_{T}$ the norm of $L^{2}\left(0, T ; L^{2}(\Omega)\right)$ :

$$
|u|_{T}=\left(\sum_{n=1}^{N} \int_{I_{n}}|u|^{2} \mathrm{~d} t\right)^{1 / 2}=\left(\sum_{n=1}^{N} \int_{I_{n}} \int_{\Omega} u(x, t)^{2} \mathrm{~d} t \mathrm{~d} x\right)^{1 / 2}
$$

The following error estimate holds.

Theorem 3.1. Under the assumption (2.4), the error e given by (3.1) satisfies

$$
|e|_{T} \leq E_{0}\left(u_{0}, M_{0}\right)+E(V, W, M, m, K, k)
$$

where

$$
E_{0}\left(u_{0}, M_{0}\right)=\sqrt{\mu_{1}}\left|\left(I-P_{M_{0}}\right) u_{0}\right|,
$$




$$
\begin{aligned}
E(V, W, M, m, K, k)= & \frac{\sqrt{\mu_{2}}}{\nu}\left|\frac{\left(P_{m}-I\right) R_{1}}{m^{2}}\right|_{T}+\frac{\sqrt{\mu_{2}}}{\nu}\left|\frac{\left(P_{M}-I\right) R_{1}^{\prime}}{M^{2}}\right|_{T} \\
& +\sqrt{\mu_{3}}\left|k R_{1}\right|_{T}+\sqrt{\mu_{1} T}\left|k R_{2}\right|_{T}+\sqrt{\mu_{3}}\left|k R_{3}\right|_{T}+\sqrt{\mu_{1} T}\left|k R_{4}\right|_{T} \\
& +\sqrt{\mu_{3}}\left|K R_{1}^{\prime}\right|_{T}+\sqrt{\mu_{3}}\left|K R_{2}^{\prime}\right|_{T}+\sqrt{\mu_{1} T}\left|K R_{3}^{\prime}\right|_{T}+\sqrt{\mu_{1} T}\left|K R_{4}^{\prime}\right|_{T}+\sqrt{\mu_{1} T}\left|K R_{5}\right|_{T}
\end{aligned}
$$

Here $R_{1}, R_{1}^{\prime}, R_{2}, R_{2}^{\prime}, R_{3}, R_{3}^{\prime}, R_{4}, R_{4}^{\prime}, R_{5}$ are defined on $I_{n}\left(I_{n} \subset J_{q}\right)$ by:

$$
\begin{aligned}
& R_{1}=\frac{V_{n}-V_{n-1}}{k_{n}}-P_{m_{q}} f+P_{m_{q}}\left(a \cdot \nabla\left(V_{n}+W_{q}\right)\right), \\
& R_{1}^{\prime}=\frac{W_{q}-W_{q-1}}{K_{q}}-\left(I-P_{m_{q}}\right) f+\left(I-P_{m_{q}}\right)\left(a \cdot \nabla\left(V_{n}+W_{q}\right)\right), \\
& R_{2}=\frac{1}{k_{n}}\left(\frac{V_{n}-P_{m_{q}}\left(\left(V_{n-1}+W_{q-1}\right)\left(X_{n-1}\right)\right)}{k_{n}}\right. \\
& \left.-P_{m_{q}}\left(a . \nabla\left(V_{n}+W_{q}\right)\right)-\frac{V_{n}-V_{n-1}}{k_{n}}\right), \\
& R_{2}^{\prime}=\frac{1}{K_{Q}}\left(\frac{W_{n}-\left(P_{M_{q}}-P_{m_{q}}\right)\left(\left(V_{n_{q-1}}+W_{q-1}\right)\left(Y_{q-1}\right)\right)}{K_{q}}\right. \\
& \left.-\left(I-P_{m_{q}}\right)\left(a \cdot \nabla\left(V_{n}+W_{q}\right)\right)-\frac{W_{q}-W_{q-1}}{K_{q}}\right), \\
& R_{3}=\frac{V_{n}-V_{n-1}}{k_{n}}, R_{3}^{\prime}=\frac{W_{q}-W_{q-1}}{K_{q}} \\
& R_{4}=\frac{p_{m_{q}}\left(f(t)-f\left(t_{n}\right)\right)}{k_{n}}, R_{4}^{\prime}=\frac{\left(I-P_{m_{q}}\right)\left(f(t)-f\left(T_{q}\right)\right)}{K_{q}}, \\
& R_{5}=\frac{\left(P_{m_{q}}-P_{m_{q-1}}\right) W_{q-1}}{K_{q}^{2}} .
\end{aligned}
$$

The constants $\mu_{1}, \mu_{2}, \mu_{3}$ depend only on $T, \nu$ and $a$.

Remark 3.1. Thanks to techniques similar to the ones in the proof of theorem 3.1 below, an a posteriori error estimate for the one-level algorithm (2.7) can be derived. Let $U(t)=U_{n}, M(t)=M_{n}$, and $k(t)=k_{n}$ for $t \in I_{n}$. Then, under assumption (2.4), the following estimate holds

$$
|u-U|_{T} \leq \mathcal{E}_{0}\left(u_{0}, M_{0}\right)+\mathcal{E}(U, M, k)
$$

where

$$
\begin{aligned}
\mathcal{E}_{0}\left(u_{0}, M_{0}\right)= & \sqrt{\gamma_{1}}\left|\left(I-P_{M_{0}}\right) u_{0}\right| \\
\mathcal{E}(U, M, k)= & \frac{\sqrt{\gamma_{2}}}{\nu}\left|\frac{\left(P_{M_{n}}-I\right) \mathcal{R}_{1}}{M^{2}}\right|_{T}+\sqrt{\gamma_{3}}\left|k \mathcal{R}_{1}\right|_{T}+\sqrt{\gamma_{1} T}\left|k \mathcal{R}_{2}\right|_{T} \\
& +\sqrt{\gamma_{3}}\left|k \mathcal{R}_{3}\right|_{T}+\sqrt{\gamma_{1} T}\left|k \mathcal{R}_{4}\right|_{T}
\end{aligned}
$$


Moreover

$$
\begin{gathered}
\mathcal{R}_{1 \mid I_{n}}=\frac{U_{n}-U_{n-1}}{k_{n}}+a . \nabla U_{n}-f(t), \\
\mathcal{R}_{2 \mid I_{n}}=\frac{1}{k_{n}}\left(\frac{U_{n}-U_{n-1}\left(X_{n-1}\right)}{k_{n}}-a . \nabla U_{n}-\frac{U_{n}-U_{n-1}}{k_{n}}\right), \\
\mathcal{R}_{3 \mid I_{n}}=\frac{U_{n}-U_{n-1}}{k_{n}}, \\
\mathcal{R}_{4 \mid I_{n}}=\frac{f(t)-f\left(t_{n}\right)}{k_{n}} .
\end{gathered}
$$

The constants $\gamma_{1}, \gamma_{2}, \gamma_{3}$ depend only on $T, \nu$ and $a$.

Here, $\mathcal{R}_{1}$ represents some residual term on the equation while $\mathcal{R}_{2}$ represents the error in the approximation of the time derivative along the characteristic curves. The term $\mathcal{R}_{3}$ corresponds to a discrete time derivative of $U_{n}$ while $\mathcal{R}_{4}$ represents some residual term related to the forcing term.

Remark 3.2. The constants $\mu_{1}, \mu_{2}, \mu_{3}$ in (3.3) and the constants $\gamma_{1}, \gamma_{2}, \gamma_{3}$ in (3.12) are stability constants related to appropriate backward dual problems. Some remarks on their computation can be found in Section 4.2.

Remark 3.3. The terms $R_{1}, R_{2}, R_{3}, R_{4}, R_{1}^{\prime}, R_{2}^{\prime}, R_{3}^{\prime}, R_{4}^{\prime}$ in the estimate (3.3) can be interpreted as above for the one-level method (Remark 3.1). They correspond respectively to the $V$ and $W$ equations. The term $R_{5}$ can be viewed as a de-refine term. It vanishes if $m_{q}<m_{q-1}$.

\section{Proof of theorem 3.1}

The proof consists in the following steps:

a) Introduction of a backward dual problem and strong stability estimates for this problem.

b) Representation of the error in terms of the residual of the approximate solution and the solution of the dual problem.

c) Projection error estimates for the solution of the dual problem.

d) Conclusion of the proof.

a) The dual problem.

The error $e$ is defined by (3.1). We introduce the following backward dual problem:

$$
\left\{\begin{array}{l}
-\frac{\partial \psi}{\partial t}-a \cdot \nabla \psi-\nu \Delta \psi=e \\
\psi(T)=0 \\
\psi \text { is } \Omega \text {-periodic. }
\end{array}\right.
$$

The following lemma provides strong stability estimates for this problem.

Lemma 3.1. Let $\psi$ be the solution of (3.17). Then, there exist constants $\mu_{1}(T), \mu_{2}(T, \nu, a)$ and $\mu_{3}(T, \nu, a)$ such that:

$$
|\psi|_{L^{\infty}\left(0, T ; L^{2}(\Omega)\right)}^{2} \leq \mu_{1}|e|_{T}^{2}
$$




$$
\begin{gathered}
\nu^{2}|\Delta \psi|_{T}^{2} \leq \mu_{2}|e|_{T}^{2}, \\
\left|\frac{\partial \psi}{\partial t}\right|_{T}^{2} \leq \mu_{3}|e|_{T}^{2} .
\end{gathered}
$$

Proof of lemma 3.1. By taking the $L^{2}$ scalar product of (3.17) with $\psi$ we have

$$
-\frac{1}{2} \frac{\mathrm{d}}{\mathrm{d} t}|\psi|^{2}-(a . \nabla \psi, \psi)-\nu(\Delta \psi, \psi)=(e, \psi)
$$

Using the periodic boundary conditions, we obtain since $a$ is divergence free that:

$$
(a . \nabla \psi, \psi)=-(\psi, a . \nabla \psi) \text { and }(a . \nabla \psi, \psi)=0
$$

Then, (3.21) becomes

$$
-\frac{1}{2} \frac{\mathrm{d}}{\mathrm{d} t}|\psi|^{2}+\nu|\nabla \psi|^{2} \leq|e||\psi| \leq \frac{1}{2}|e|^{2}+\frac{1}{2}|\psi|^{2}
$$

which provides by integration on $[t, T]$

$$
|\psi(t)|^{2}+2 \nu \int_{t}^{T}|\nabla \psi(s)|^{2} \mathrm{~d} s \leq|e|_{T}^{2}+\int_{t}^{T}|\psi(s)|^{2} \mathrm{~d} s .
$$

Using Gronwall lemma this gives that

$$
|\psi(t)|^{2}+2 \nu \int_{t}^{T}|\nabla(s)|^{2} \mathrm{~d} s \leq e^{T-t}|e|_{T}^{2}
$$

that is $(3.18)$ with $\mu_{1}=e^{T}$. Also this inequality for $t=0$ provides that

$$
|\psi(0)|^{2}+2 \nu|\nabla \psi|_{T}^{2} \leq e^{T}|e|_{T}^{2}
$$

Now, taking the scalar product of (3.17) with $-\nu \Delta \psi$ we have that

$$
-\frac{1}{2} \frac{\mathrm{d}}{\mathrm{d} t} \nu|\nabla \psi|^{2}+\nu^{2}|\Delta \psi|^{2} \leq|(e+a . \nabla \psi, \nu \Delta \psi)| \leq \frac{1}{2} \nu^{2}|\Delta \psi|^{2}+|e|^{2}+|a . \nabla \psi|^{2},
$$

hence

$$
-\frac{\mathrm{d}}{\mathrm{d} t} \nu|\nabla \psi|^{2}+\nu^{2}|\Delta \psi|^{2} \leq 2|e|^{2}+2|a|_{L^{\infty}\left(0, T ; L^{\infty}(\Omega)\right)}^{2}|\nabla \psi|^{2}
$$

By integration of (3.24) on $[t, T]$ we have that

$$
\nu|\nabla \psi(t)|^{2}+\int_{t}^{T} \nu^{2}|\Delta \psi(s)|^{2} \mathrm{~d} s \leq 2|e|_{T}^{2}+2|a|_{L^{\infty}\left(0, T ; L^{\infty}(\Omega)\right)}^{2} \int_{t}^{T}|\nabla \psi(s)|^{2} \mathrm{~d} s
$$

and

$$
\nu|\nabla \psi(t)|^{2}+\int_{t}^{T} \nu^{2}|\Delta \psi(s)|^{2} \mathrm{~d} s \leq 2|e|_{T}^{2} e^{\frac{2|a|_{L}^{2} \infty(0, T ; L \infty(\Omega))}{\nu}(T-t)} .
$$


This inequality for $t=0$ yields (3.19).

Finally, taking the scalar product of (3.17) with $-\frac{\partial \psi}{\partial t}$ we have that

$$
\begin{aligned}
\left|\frac{\partial \psi}{\partial t}\right|^{2}-\frac{1}{2} \frac{\mathrm{d}}{\mathrm{d} t} \nu|\nabla \psi|^{2} & \leq\left|\left(a . \nabla \psi, \frac{\partial \psi}{\partial t}\right)\right|+\left|\left(e, \frac{\partial \psi}{\partial t}\right)\right| \\
& \leq|a|_{L^{\infty}\left(0, T ; L^{\infty}(\Omega)\right)}|\nabla \psi|\left|\frac{\partial \psi}{\partial t}\right|+|e|\left|\frac{\partial \psi}{\partial t}\right| \\
& \leq \frac{1}{2}\left|\frac{\partial \psi}{\partial t}\right|^{2}+|a|_{L^{\infty}\left(0, T ; L^{\infty}(\Omega)\right)}^{2}|\nabla \psi|^{2}+|e|^{2},
\end{aligned}
$$

hence

$$
-\frac{\mathrm{d}}{\mathrm{d} t} \nu|\nabla \psi|^{2}+\left|\frac{\partial \psi}{\partial t}\right|^{2} \leq 2|a|_{L^{\infty}\left(0, T ; L^{\infty}(\Omega)\right)}^{2}|\nabla \psi|^{2}+2|e|^{2} .
$$

By integration on $[0, T]$, using moreover (3.23), we obtain (3.20). This concludes the proof of lemma 3.1 .

b) Error representation.

Here we derive a representation of the norm $|e|_{T}$ of the error (where $|\cdot|_{T}$ is defined by (3.2)) that uses the solution of the dual problem (3.17).

We start by multiplying (3.17) by $e$ and integrating by parts both in space and time. This gives that

$$
\left.|e|_{T}^{2}=\sum_{n=1}^{N} \int_{I_{n}}\left(\left(\frac{\partial e}{\partial t}+a . \nabla e, \psi\right)+\nu(\nabla \psi, \nabla e)\right) \mathrm{d} t-\sum_{n=1}^{N} \int_{\Omega} e\left(t_{n}^{-}\right) \psi\left(t_{n}\right)-e\left(t_{n-1}^{+}\right) \psi\left(t_{n-1}\right)\right) \mathrm{d} x
$$

where

$$
e\left(t_{n-1}^{+}\right)=\lim _{t \rightarrow t_{n-1}^{+}} e(t)=u\left(t_{n-1}\right)-\left(V_{n}+W_{q}\right), e\left(t_{n}^{-}\right)=\lim _{t \rightarrow t_{n}^{-}} e(t)=u\left(t_{n}\right)-\left(V_{n}+W_{q}\right)
$$

This equality re-writes

$$
\begin{aligned}
|e|_{T}^{2}= & \sum_{n=1}^{N} \int_{I_{n}}\left(\left(\frac{\partial e}{\partial t}+a . \nabla e, \psi\right)+\nu(\nabla \psi, \nabla e)\right) \mathrm{d} t-\left(\left(V_{0}+W_{0}\right)-u_{0}, \psi(0)\right) \\
& -\sum_{n=1}^{N}\left(V_{n}-V_{n-1}, \psi\left(t_{n-1}\right)\right)-\sum_{q=1}^{Q}\left(W_{q}-W_{q-1}, \psi\left(T_{q-1}\right)\right) .
\end{aligned}
$$

On the time interval $I_{n}$, thanks to (3.1) and (2.1) we have that

$$
\begin{aligned}
\left(\frac{\partial e}{\partial t}+a . \nabla e, \psi\right) & =\left(f+\nu \Delta u-a . \nabla\left(V_{n}+W_{q}\right), \psi\right) \\
& =\left(f-a . \nabla\left(V_{n}+W_{q}\right), \psi\right)-\nu(\nabla u, \nabla \psi)
\end{aligned}
$$


Therefore (3.25) becomes

$$
\begin{aligned}
|e|_{T}^{2}= & \left.\sum_{n=1}^{N} \int_{I_{n}}\left(f-a \cdot \nabla\left(V_{n}+W_{q}\right), \psi\right)\right) \mathrm{d} t \\
& -\sum_{n=1}^{N} \int_{I_{n}} \nu\left(\nabla\left(V_{n}+W_{q}\right), \nabla \psi\right) \mathrm{d} t-\left(V_{0}+W_{0}-u_{0}, \psi(0)\right) \\
& -\sum_{n=1}^{N}\left(V_{n}-V_{n-1}, \psi\left(t_{n-1}\right)\right)-\sum_{q=1}^{Q}\left(W_{q}-W_{q-1}, \psi\left(T_{q-1}\right)\right) .
\end{aligned}
$$

Let us now introduce $\Psi=\Psi_{1}+\Psi_{2}$, such that $\Psi_{1 \mid J_{q}} \in S_{m_{q}}, \Psi_{2 \mid J_{q}} \in\left(I-P_{m_{q}}\right) S_{M_{q}}$. Also we assume that the value of $\Psi_{1}$ does not depend on time on $I_{n}$ while $\Psi_{2}$ does not depend on time on $J_{q}$. Taking the scalar product of $(2.8 \mathrm{a})$ and $(2.8 \mathrm{~b})$ with $\Psi$, we see that

$$
\left(\frac{V_{n}-P_{m_{q}}\left(V_{n-1}+W_{q-1}\right)\left(X_{n-1}\right)}{k_{n}}-\nu \Delta V_{n}, \Psi\right)=\left(P_{m_{q}} f\left(t_{n}\right), \Psi\right)
$$

and

$$
\left(\frac{W_{q}-\left(P_{M_{q}}-P_{m_{q}}\right)\left(V_{n_{q-1}}+W_{q-1}\right)\left(Y_{q-1}\right)}{K_{q}}-\nu \Delta W_{q}, \Psi\right)=\left(\left(P_{M_{q}}-P_{m_{q}}\right) f\left(T_{q}\right), \Psi\right)
$$

These inequalities together with (3.26) enable us to say that

$$
\begin{aligned}
|e|_{T}^{2}= & \sum_{n=1}^{N} \int_{I_{n}}\left(f-a \cdot \nabla\left(V_{n}+W_{q}\right), \psi\right) \mathrm{d} t-\sum_{n=1}^{N}\left(V_{n}-V_{n-1}, \psi\left(t_{n-1}\right)\right) \\
& -\sum_{q=1}^{Q}\left(W_{q}-W_{q-1}, \psi\left(T_{q-1}\right)\right)-\left(V_{0}+W_{0}-u_{0}, \psi(0)\right) \\
& -\sum_{n=1}^{N} \int_{I_{n}} \nu\left(\nabla\left(V_{n}+W_{q}\right), \nabla(\psi-\Psi)\right) \mathrm{d} t \\
& +\sum_{n=1}^{N} \int_{I_{n}}\left(\frac{V_{n}-P_{m_{q}}\left(V_{n-1}+W_{q-1}\right)\left(X_{n-1}\right)}{k_{n}}-P_{m_{q}} f\left(t_{n}\right), \Psi\right) \mathrm{d} t \\
& +\sum_{n=1}^{N} \int_{I_{n}}\left(\frac{W_{q}-\left(P_{M_{q}}-P_{m_{q}}\right)\left(V_{n_{q-1}}+W_{q-1}\right)\left(Y_{q-1}\right)}{K_{q}}-\left(P_{M_{q}}-P_{m_{q}}\right) f\left(T_{q}\right), \Psi\right) \mathrm{d} t .
\end{aligned}
$$

Next, we have that

$$
\begin{gathered}
\frac{V_{n}-P_{m_{q}}\left(V_{n-1}+W_{q-1}\right)\left(X_{n-1}\right)}{k_{n}}-P_{m_{q}} f\left(t_{n}\right) \in S_{m_{q}}, \\
\frac{W_{q}-\left(P_{M_{q}}-P_{m_{q}}\right)\left(V_{n_{q-1}}+W_{q-1}\right)\left(Y_{q-1}\right)}{K_{q}}-\left(P_{M_{q}}-P_{m_{q}}\right) f\left(T_{q}\right) \in\left(I-P_{m_{q}}\right) S_{M_{q}} .
\end{gathered}
$$


Thus, using the projectors $P_{m_{q}}$ and $I-P_{m_{q}}$, we obtain the following error representation (recall that $I_{n} \subset J_{q}$ ):

$$
\begin{aligned}
& |e|_{T}^{2}=\sum_{n=1}^{N} \int_{I_{n}} \nu\left(\nabla\left(V_{n}+W_{q}\right), \nabla(\Psi-\psi)\right) \mathrm{d} t \\
& +\sum_{n=1}^{N} \int_{I_{n}}\left(\frac{V_{n}-V_{n-1}}{k_{n}}+P_{m_{q}}\left(a . \nabla\left(V_{n}+W_{q}\right)\right)-P_{m_{q}} f(t), \Psi_{1}-\psi\right) \mathrm{d} t \\
& +\sum_{n=1}^{N} \int_{I_{n}}\left(\frac{W_{q}-W_{q-1}}{K_{q}}+\left(I-P_{m_{q}}\right)\left(a . \nabla\left(V_{n}+W_{q}\right)\right)\right. \\
& \left.-\left(I-P_{m_{q}}\right) f(t), \Psi_{2}-\psi\right) \mathrm{d} t \\
& +\sum_{n=1}^{N} \int_{I_{n}}\left(\frac{V_{n}-P_{m_{q}}\left(V_{n-1}+W_{q-1}\right)\left(X_{n-1}\right)}{k_{n}}-P_{m_{q}}\left(a . \nabla\left(V_{n}+W_{q}\right)\right)\right. \\
& \left.-\frac{V_{n}-V_{n-1}}{k_{n}}, \Psi_{1}\right) \mathrm{d} t \\
& +\sum_{n=1}^{N} \int_{I_{n}}\left(\frac{W_{q}-\left(P_{M_{q}}-P_{m_{q}}\right)\left(V_{n_{q-1}}+W_{q-1}\right)\left(Y_{q-1}\right)}{K_{q}}\right. \\
& \left.-\left(I-P_{m_{q}}\right)\left(a . \nabla\left(V_{n}+W_{q}\right)\right)-\frac{W_{q}-W_{q-1}}{K_{q}}, \Psi_{2}\right) \mathrm{d} t \\
& +\sum_{n=1}^{N} \int_{I_{n}}\left(\frac{V_{n}-V_{n-1}}{k_{n}}, \psi(t)-\psi\left(t_{n-1}\right)\right) \mathrm{d} t \\
& +\sum_{q=1}^{Q} \int_{J_{q}}\left(\frac{W_{q}-W_{q-1}}{K_{q}}, \psi(t)-\psi\left(T_{q-1}\right)\right) \mathrm{d} t \\
& +\left(u_{0}-\left(V_{0}+W_{0}\right), \psi(0)\right)+\sum_{n=1}^{N} \int_{I_{n}}\left(P_{m_{q}}\left(f(t)-f\left(t_{n}\right)\right), \Psi_{1}\right) \mathrm{d} t \\
& +\sum_{q=1}^{Q} \int_{J_{q}}\left(\left(I-P_{m_{q}}\right) f(t)-\left(P_{M_{q}}-P_{m_{q}}\right) f\left(T_{q}\right), \Psi_{2}\right) \mathrm{d} t .
\end{aligned}
$$

c) Projection estimates for the dual problem.

Note that the spatial projectors $P_{m}$ and $P_{M}$ defined by

$$
P_{m} v_{\mid I_{n}}=P_{m_{q}} v, P_{M} v_{\mid J_{q}}=P_{M_{q}} v, I_{n} \subset J_{q} .
$$

satisfy the following orthogonality properties:

$$
\begin{aligned}
& \text { for } v \in S_{m_{q}}, u \in H_{p}^{1}(\Omega),\left(u-P_{m_{q}} u, v\right)=\left(\nabla\left(u-P_{m_{q}} u\right), \nabla v\right)=0 \\
& \text { for } v \in S_{M_{q}}, u \in H_{p}^{1}(\Omega),\left(u-P_{M_{q}} u, v\right)=\left(\nabla\left(u-P_{M_{q}} u\right), \nabla v\right)=0 .
\end{aligned}
$$

Also the following approximation properties hold:

$$
\text { for } u \in H_{p}^{\alpha}(\Omega), \alpha \geq 0,\left|\left(I-P_{m_{q}}\right) u\right| \leq \frac{1}{m_{q}^{\alpha}}\|u\|_{\alpha},\left|\left(I-P_{M_{q}}\right) u\right| \leq \frac{1}{M_{q}^{\alpha}}\|u\|_{\alpha}
$$




$$
\text { for } u \in H_{p}^{2}(\Omega),\left|\left(I-P_{m_{q}}\right) u\right| \leq \frac{1}{m_{q}^{2}}|\Delta u|,\left|\left(I-P_{M_{q}}\right) u\right| \leq \frac{1}{M_{q}^{2}}|\Delta u| \text {. }
$$

Properties (3.31) are easily deduced from (3.30) by using that the functions $\left(I-P_{m_{q}}\right) u$ and $\left(I-P_{M_{q}}\right) u$ have zero mean value.

Next, we introduce some temporal projections. For $u \in L^{1}\left(I_{n} ; H_{p}^{\alpha}(\Omega)\right)$ we define $\pi_{n} u$ by

$$
\pi_{n} u=\frac{1}{k_{n}} \int_{I_{n}} u(t) \mathrm{d} t
$$

Then for $u \in L^{1}\left(0, T ; H_{p}^{\alpha}(\Omega)\right)$, we set

$$
\pi u_{\mid I_{n}}=\pi_{n} u
$$

Similarly we write:

$$
\Pi v_{\mid J_{q}}=\Pi_{q} v=\frac{1}{K_{q}} \int_{J_{q}} v(t) \mathrm{d} t
$$

It is easily checked that the projectors $\pi$ and $\Pi$ satisfy the following orthogonality and approximation properties:

$$
\begin{gathered}
\text { for } u \in L^{1}\left(I_{n} ; H_{p}^{1}(\Omega)\right), v \in H_{p}^{1}(\Omega), \int_{I_{n}}\left(u-\pi_{n} u, v\right) \mathrm{d} t=\int_{I_{n}}\left(\nabla\left(u-\pi_{n} u\right), \nabla v\right) \mathrm{d} t=0 \\
\text { for } u \in L^{1}\left(J_{q} ; H_{p}^{1}(\Omega)\right), v \in H_{p}^{1}(\Omega), \int_{J_{q}}\left(u-\Pi_{q} u, v\right) \mathrm{d} t=\int_{J_{q}}\left(\nabla\left(u-\Pi_{q} u\right), \nabla v\right) \mathrm{d} t=0 \\
\qquad \text { for } u \in L^{2}\left(0, T ; L^{2}(\Omega)\right) \text { such that } \frac{\partial u}{\partial t} \in L^{2}\left(0, T ; L^{2}(\Omega)\right), \\
\qquad\left|\frac{(\pi-I) u}{k}\right|_{T} \leq\left|\frac{\partial u}{\partial t}\right|_{T},\left|\frac{(\Pi-I) u}{K}\right|_{T} \leq\left|\frac{\partial u}{\partial t}\right|_{T}
\end{gathered}
$$

With these notations, the choice of $\Psi$ in (3.27) is as follows

$$
\Psi=\Psi_{1}+\Psi_{2}
$$

with

$$
\Psi_{1}=P_{m} \pi \psi=\pi P_{m} \psi, \Psi_{2}=\left(P_{M}-P_{m}\right) \Pi \psi=\Pi\left(P_{M}-P_{m}\right) \psi
$$

d) Conclusion of the proof of theorem 3.1.

We now proceed to estimate the right hand side of (3.27) with the above choice of $\Psi$. We will use the residual notations (3.4)-(3.10). 
For the first term we have

$$
\begin{aligned}
& \sum_{n=1}^{N} \int_{I_{n}} \nu\left(\nabla\left(V_{n}+W_{q}\right), \nabla(\Psi-\psi)\right) \mathrm{d} t= \\
& \sum_{n=1}^{N} \int_{I_{n}} \nu\left(\nabla\left(V_{n}+W_{q}\right), \nabla P_{m_{q}}\left(\pi_{n}-I\right) \psi\right) \mathrm{d} t \\
& +\sum_{n=1}^{N} \int_{I_{n}} \nu\left(\nabla\left(V_{n}+W_{q}\right), \nabla\left(P_{M_{q}}-I\right) \psi\right) \mathrm{d} t \\
& +\sum_{n=1}^{N} \int_{I_{n}} \nu\left(\nabla\left(V_{n}+W_{q}\right), \nabla\left(P_{M_{q}}-P_{m_{q}}\right)\left(\Pi_{q}-I\right) \psi\right) \mathrm{d} t .
\end{aligned}
$$

Since

$$
\begin{aligned}
& \left(\nabla W_{q}, \nabla P_{m_{q}}\left(\pi_{n}-I\right) \psi\right)=0,\left(\nabla V_{n}, \nabla\left(P_{M_{q}}-I\right) \psi\right)=0, \\
& \left(\nabla V_{n}, \nabla\left(P_{M_{q}}-P_{m_{q}}\right)\left(\Pi_{q}-I\right) \psi\right)=0,
\end{aligned}
$$

we have by using also orthogonality properties

$$
\sum_{n=1}^{N} \int_{I_{n}} \nu\left(\nabla\left(V_{n}+W_{q}\right), \nabla(\Psi-\psi)\right) \mathrm{d} t=0
$$

The second term is

$$
\sum_{n=1}^{N} \int_{I_{n}}\left(R_{1}, \Psi_{1}-\psi\right) \mathrm{d} t
$$

We re-write $\Psi_{1}-\psi$ as $\left(P_{m_{q}}-I\right) \psi+\left(\pi_{n}-I\right) P_{m_{q}} \psi$, and use properties (3.31) and (3.36). This gives that

$$
\begin{aligned}
\left|\sum_{n=1}^{N} \int_{I_{n}}\left(R_{1}, \Psi_{1}-\psi\right) \mathrm{d} t\right| & \leq\left|k R_{1}\right|_{T}\left|\frac{(\pi-I) P_{m} \psi}{k}\right|_{T}+\left|\frac{\left(P_{m}-I\right) R_{1}}{m^{2}}\right|_{T}\left|m^{2}\left(P_{m}-I\right) \psi\right|_{T} \\
& \leq\left|k R_{1}\right|_{T}\left|\frac{\partial \psi}{\partial t}\right|_{T}+\left|\frac{\left(P_{m}-I\right) R_{1}}{m^{2}}\right|_{T}|\Delta \psi|_{T} .
\end{aligned}
$$

Now, applying the stability results of Lemma 3.1, we obtain that

$$
\left|\sum_{n=1}^{N} \int_{I_{n}}\left(R_{1}, \Psi_{1}-\psi\right) \mathrm{d} t\right| \leq\left(\sqrt{\mu_{3}}\left|k R_{1}\right|_{T}+\frac{\sqrt{\mu_{2}}}{\nu}\left|\frac{\left(P_{m}-I\right) R_{1}}{m^{2}}\right|_{T}\right)|e|_{T} .
$$

Next, for the third term, we split $\Psi_{2}-\psi$ as follows

$$
\Psi_{2}-\psi=\left(P_{M_{q}} \Pi_{q} \psi-\psi\right)-P_{m_{q}} \Pi_{q} \psi .
$$

We need to estimate

$$
\left|\sum_{n=1}^{N} \int_{I_{n}}\left(R_{1}^{\prime}, P_{M_{q}} \Pi_{q} \psi-\psi\right) \mathrm{d} t-\sum_{n=1}^{N} \int_{I_{n}}\left(R_{1}^{\prime}, P_{m_{q}} \Pi_{q} \psi\right) \mathrm{d} t\right| .
$$


The first term of (3.40) is bounded thanks to computations similar to the ones before (3.38) and (3.39). We see that

$$
\left|\sum_{n=1}^{N} \int_{I_{n}}\left(R_{1}^{\prime}, P_{M_{q}} \Pi_{q} \psi-\psi\right) \mathrm{d} t\right| \leq\left(\sqrt{\mu_{3}}\left|K_{q} R_{1}^{\prime}\right|_{T}+\frac{\sqrt{\mu_{2}}}{\nu}\left|\frac{\left(P_{M_{q}}-I\right) R_{1}^{\prime}}{M_{q}^{2}}\right|_{T}\right)|e|_{T} .
$$

For the second term of (3.40) we have

$$
\begin{aligned}
-\sum_{n=1}^{N} \int_{I_{n}}\left(R_{1}^{\prime}, P_{m_{q}} \Pi_{q} \psi\right) \mathrm{d} t & =\sum_{n=1}^{N} \int_{I_{n}}\left(\frac{W_{q-1}}{K_{q}}, P_{m_{q}} \Pi_{q} \psi\right) \mathrm{d} t \\
& =\sum_{n=1}^{N} \int_{I_{n}}\left(\frac{\left(P_{m_{q}}-P_{m_{q-1}}\right) W_{q-1}}{K_{q}}, P_{m_{q}} \Pi_{q} \psi\right) \mathrm{d} t .
\end{aligned}
$$

Note that, if $m_{q}<m_{q-1}$ (that is $W$ is de-refined), this term is equal to zero. By using Lemma 3.1, we estimate that term as follows

$$
\begin{aligned}
\left|\sum_{n=1}^{N} \int_{I_{n}}\left(K_{q} R_{5}, P_{m_{q}} \Pi_{q} \psi\right) \mathrm{d} t\right| & \leq\left|K_{q} R_{5}\right|_{T}\left|P_{m_{q}} \Pi_{q} \Psi\right|_{T} \\
& \leq\left|K_{q} R_{5}\right|_{T}|\Psi|_{T} \leq\left|K_{q} R_{5}\right|_{T}|\psi|_{T} \\
& \leq \sqrt{T}\left|K_{q} R_{5}\right|_{T}|\psi|_{L^{\infty}\left(0, T ; L^{2}(\Omega)\right)} \\
& \leq \sqrt{\mu_{1} T}\left|K_{q} R_{5}\right|_{T}|e|_{T} .
\end{aligned}
$$

Next for the fourth term we have

$$
\sum_{n=1}^{N} \int_{I_{n}}\left(k_{n} R_{2}, \Psi_{1}\right) \mathrm{d} t \leq\left|k_{n} R_{2}\right|_{T}|\psi|_{T} \leq \sqrt{T}\left|k_{n} R_{2}\right|_{T}|\psi|_{L^{\infty}\left(0, T ; L^{2}(\Omega)\right)},
$$

and thanks to (3.18) we obtain that

$$
\left|\sum_{n=1}^{N} \int_{I_{n}}\left(k_{n} R_{2}, \Psi_{1}\right) \mathrm{d} t\right| \leq \sqrt{\mu_{1}} T\left|k_{n} R_{2}\right|_{T}|e|_{T} .
$$

Similarly, the fifth term gives

$$
\left|\sum_{n=1}^{N} \int_{I_{n}}\left(K_{q} R_{2}^{\prime}, \Psi_{2}\right) \mathrm{d} t\right| \leq \sqrt{\mu_{1}} T\left|K R_{2}^{\prime}\right|_{T}|e|_{T}
$$

For the sixth term, using (3.20), we have

$$
\begin{aligned}
\left|\sum_{n=1}^{N} \int_{I_{n}}\left(R_{3}, \psi(t)-\psi\left(t_{n-1}\right)\right) \mathrm{d} t\right| & \leq\left|k R_{3}\right|_{T}\left(\sum_{n=1}^{N} \frac{1}{k_{n}^{2}} \int_{I_{n}}\left|\psi(t)-\psi\left(t_{n-1}\right)\right|^{2} \mathrm{~d} t\right)^{1 / 2} \\
& \leq\left|k R_{3}\right|_{T}\left(\sum_{n=1}^{N} \int_{I_{n}}\left|\frac{\partial \psi}{\partial t}(s)\right|^{2} \mathrm{~d} s\right)^{1 / 2} \\
& \leq\left|k R_{3}\right|_{T}\left|\frac{\partial \psi}{\partial t}\right|_{T} \leq \sqrt{\mu_{3}}\left|k R_{3}\right|_{T}|e|_{T} .
\end{aligned}
$$


Similarly, the seventh term gives

$$
\left|\sum_{q=1}^{Q} \int_{J_{q}}\left(R_{3}^{\prime}, \psi(t)-\psi\left(T_{q-1}\right)\right) \mathrm{d} t\right| \leq \sqrt{\mu_{3}}\left|K R_{3}^{\prime}\right|_{T}|e|_{T} .
$$

The eighth term corresponds to the part $E_{0}$ of the estimate. We have

$$
\left|\left(u_{0}-\left(V_{0}+W_{0}\right), \psi(0)\right)\right| \leq \sqrt{\mu_{1}}\left|\left(I-P_{M_{0}}\right) u_{0}\right||e|_{T} .
$$

Next, the ninth term of (3.27) is estimated with computations similar to the ones before. We have

$$
\left|\sum_{n=1}^{N} \int_{I_{n}}\left(k_{n} R_{4}, \Psi_{1}\right) \mathrm{d} t\right| \leq \sqrt{\mu_{1} T}\left|k R_{4}\right|_{T}|e|_{T} .
$$

Finally, we split the last term of (3.27) as follows:

$$
\left(I-P_{m_{q}}\right) f(t)-\left(P_{M_{q}}-P_{m_{q}}\right) f\left(T_{q}\right)=\left(I-P_{m_{q}}\right)\left(f(t)-f\left(T_{q}\right)\right)+\left(I-P_{M_{q}}\right) f\left(T_{q}\right) .
$$

We have

$$
\sum_{q=1}^{Q} \int_{J_{q}}\left(\left(I-P_{M_{q}}\right) f\left(T_{q}\right), \Psi_{2}\right) \mathrm{d} t=0 .
$$

So, we only need to estimate the term

$$
\sum_{q=1}^{Q} \int_{J_{q}}\left(\left(I-P_{m_{q}}\right)\left(f(t)-f\left(T_{q}\right)\right), \Psi_{2}\right) \mathrm{d} t .
$$

Thanks to (3.18) we obtain that

$$
\left|\sum_{q=1}^{Q} \int_{J_{q}}\left(K_{q} R_{4}^{\prime}, \Psi_{2}\right) \mathrm{d} t\right| \leq \sqrt{\mu_{1} T}\left|K R_{4}^{\prime}\right|_{T}|e|_{T}
$$

Bringing together (3.37), (3.39), (3.41), (3.43), (3.44), (3.45), (3.46), (3.47), (3.48), (3.49), (3.50) and (3.27) leads to the a posteriori estimate (3.3). The proof of Theorem 3.1 is complete.

\section{AdAptive Algorithms}

In this section, we describe an adaptive algorithm relying on the a posteriori error estimate (3.3). Since we aim to compare this algorithm with the one-level method (2.7), we first present an adaptive algorithm for (2.7). 


\subsection{The one-level adaptive algorithm}

Let $\mathcal{T O} \mathcal{L}$ be a given tolerance. Thanks to the a posteriori estimate (3.11) we design an adaptive algorithm which guarantees that

$$
|u-U|_{T} \leq \mathcal{E}_{0}\left(u_{0}, M_{0}\right)+\mathcal{E}(U, M, k) \leq \mathcal{T} \mathcal{O} \mathcal{L}
$$

where $U$ is the solution of (2.7). Note that the first term in (4.1) is easily controlled by an appropriate choice of $M_{0}$. Hence, the question is to find discretization parameters such that

$$
\mathcal{E}(U, M, k) \leq \mathcal{T} \mathcal{O} \mathcal{L}^{\prime} \approx \mathcal{T} \mathcal{O} \mathcal{L}
$$

The quantity $\mathcal{E}$ naturally splits into the sum of a term $\mathcal{E}_{M}$ that controls the spatial discretization and a term $\mathcal{E}_{k}$ that controls the temporal discretization with

$$
\begin{gathered}
\mathcal{E}_{M}(U, M, k)=\frac{4 \sqrt{\gamma_{2}}}{\nu}\left|\frac{\left(P_{M_{n}}-I\right) \mathcal{R}_{1}}{M^{2}}\right|_{T}, \\
\mathcal{E}_{k}(U, M, k)=\sqrt{\gamma_{3}}\left|k \mathcal{R}_{1}\right|_{T}+\sqrt{\gamma_{1} T}\left|k \mathcal{R}_{2}\right|_{T}+\sqrt{\gamma_{3}}\left|k \mathcal{R}_{3}\right|_{T}+\sqrt{\gamma_{1} T}\left|k \mathcal{R}_{4}\right|_{T} .
\end{gathered}
$$

It is easily seen that

$$
\mathcal{E}_{M}(U, M, k) \leq \max _{1 \leq n \leq N} \frac{\mathcal{E}_{n}^{\prime}}{M_{n}^{2}}, \mathcal{E}_{k}(U, M, k) \leq \max _{1 \leq n \leq N} k_{n} \mathcal{E}_{n}^{\prime \prime}
$$

where

$$
\mathcal{E}_{n}^{\prime}=\frac{4 \sqrt{\gamma_{2} T}}{\nu}\left|\left(P_{M_{n}}-I\right) \mathcal{R}_{1 \mid I_{n}}\right|,|.|=\text { norm of } L^{2}(\Omega)
$$

and

$$
\mathcal{E}_{n}^{\prime \prime}=\sqrt{\gamma_{3} T}\left|\mathcal{R}_{1 \mid I_{n}}\right|+\sqrt{\gamma_{1}} T\left|\mathcal{R}_{2 \mid I_{n}}\right|+\sqrt{\gamma_{3} T}\left|\mathcal{R}_{3 \mid I_{n}}\right|+\sqrt{\gamma_{1}} T\left|\mathcal{R}_{4 \mid I_{n}}\right|
$$

This leads us to require that

$$
\max _{1 \leq n \leq N} \frac{\mathcal{E}_{n}^{\prime}}{M_{n}^{2}} \leq \mathcal{T} \mathcal{O} \mathcal{L}_{M}, \text { and } \max _{1 \leq n \leq N} k_{n} \mathcal{E}_{n}^{\prime \prime} \leq \mathcal{T} \mathcal{O} \mathcal{L}_{k}
$$

where $\mathcal{T} \mathcal{O} \mathcal{L}^{\prime}=\mathcal{T} \mathcal{O} \mathcal{L}_{M}+\mathcal{T} \mathcal{O} \mathcal{L}_{k}$

At this point, choosing the parameter $\mathcal{T} \mathcal{O} \mathcal{L}_{M}=\mathcal{T} \mathcal{O} \mathcal{L}_{k}=\mathcal{T} \mathcal{O} \mathcal{L} / 2$ could seem natural. However since the parameter $M_{n}$ is discrete, this choice would often lead to $\mathcal{E}_{M} \ll \mathcal{T O} \mathcal{L} / 2$, so that the estimated error would be about half the tolerance which implies a loss of efficiency for the algorithm. Therefore, we will set

$$
\mathcal{T} \mathcal{O} \mathcal{L}_{M}=\frac{1}{10} \mathcal{T} \mathcal{O} \mathcal{L} \text { and } \mathcal{T} \mathcal{O} \mathcal{L}_{k}=\frac{9}{10} \mathcal{T} \mathcal{O} \mathcal{L}
$$

The adaptive algorithm proceeds as follows.

- $M_{0}$ is chosen so that $\mathcal{E}_{0}\left(u_{0}, M_{0}\right) \ll \mathcal{T} \mathcal{O} \mathcal{L}$. 
- At a typical iteration $n$, the parameters $k_{n}, M_{n}$ and the approximate solution $U_{n}$ are determined by requiring that

$$
\frac{\mathcal{E}_{n}^{\prime}}{M_{n}^{2}} \approx \mathcal{T} \mathcal{O} \mathcal{L}_{M} \text { and } k_{n} \mathcal{E}_{n}^{\prime \prime} \approx \mathcal{T} \mathcal{O} \mathcal{L}_{k}
$$

Note that $\mathcal{E}_{n}^{\prime}$ and $\mathcal{E}_{n}^{\prime \prime}$ depend on $\left(U_{n-1}, M_{n-1}, k_{n-1}\right)$ and $\left(U_{n}, M_{n}, k_{n}\right)$. Therefore the conditions (4.4) are reached thanks to some iterative procedure yielding $\left(k_{n}, M_{n}, U_{n}\right)$. More precisely, we introduce a sequence $\left(k_{n}^{j}, M_{n}^{j}, U_{n}^{j}\right)_{j \geq 0}$ that tends to $\left(k_{n}, M_{n}, U_{n}\right)$ satisfying (4.4) as follows. Suppose that $\left(k_{n-1}, M_{n-1}, U_{n-1}\right)$ is known. We set $k_{n}^{0}=k_{n-1}, M_{n}^{0}=M_{n-1}$ and $U_{n}^{0}$ is the corresponding solution of (2.7a). Then, for $j \geq 1$, $\left(k_{n}^{j}, M_{n}^{j}, U_{n}^{j}\right)$ are defined as follows

. $k_{n}^{j}$ is the solution of $k_{n}^{j} \mathcal{E}_{n}^{\prime \prime}\left(k_{n}^{j-1}, M_{n}^{j-1}, U_{n}^{j-1}\right)=\mathcal{T} \mathcal{O} \mathcal{L}_{k}$;

. $M_{n}^{j}$ is the smallest integer $M$ satisfying $\frac{1}{M^{2}} \mathcal{E}_{n}^{\prime}\left(k_{n}^{j-1}, M, U_{n}^{j-1}\right)=\mathcal{T} \mathcal{O} \mathcal{L}_{M}$;

. $U_{n}^{j}$ is the solution of $U_{n}^{j}-P_{M_{n}^{j-1}} U_{n-1}\left(X_{n-1}\right)-k_{n}^{j-1} \nu \Delta U_{n}^{j}=k_{n}^{j-1} P_{M_{n}^{j-1}} f\left(t_{n}\right)$.

The procedure is re-iterated till reaching the stopping condition $\mathcal{E}\left(k_{n}^{j}, M_{n}^{j}, U_{n}^{j}\right) \leq \mathcal{T} \mathcal{O} \mathcal{L}$. Note that it converges in one or two iterations.

Some remarks on the estimation of the stability constants $\gamma_{i}, i=1,2,3$ can be found in Section 4.2.

\subsection{The two-level adaptive algorithm}

Here, thanks to the estimate (3.3), we define an adaptive algorithm for the scheme (2.8) that guarantees that

$$
|u-(V+W)|_{T} \leq E_{0}\left(u_{0}, M_{0}\right)+E(V, W, M, m, K, k) \leq T O L
$$

where $T O L$ is some given tolerance. We first note that

$$
E(V, W, M, m, K, k) \leq \max _{1 \leq q \leq Q} \frac{E_{q}^{\prime}}{M_{q}^{2}}+\max _{1 \leq q \leq Q} \frac{E_{q}^{\prime \prime}}{m_{q}^{2}}+\max _{1 \leq q \leq Q} K_{q} E_{q}^{\prime \prime \prime}+\max _{1 \leq n \leq N} k_{n} E_{n}
$$

where

$$
\begin{gathered}
E_{q}^{\prime}=\frac{4 \sqrt{\mu_{2} T}}{\nu}\left|\left(P_{M_{q}}-I\right) R_{1}^{\prime}\right|, E_{q}^{\prime \prime}=\frac{4 \sqrt{\mu_{2} T}}{\nu}\left|\left(P_{m_{q}}-I\right) R_{1}\right|, \\
E_{q}^{\prime \prime \prime}=\sqrt{\mu_{3} T}\left|R_{1}^{\prime}\right|+\sqrt{\mu_{1}} T\left|R_{2}^{\prime}\right|+\sqrt{\mu_{3} T}\left|R_{3}^{\prime}\right|+\sqrt{\mu_{1}} T\left|R_{4}^{\prime}\right|+\sqrt{\mu_{1}} T\left|R_{5}\right|
\end{gathered}
$$

and

$$
E_{n}=\sqrt{\mu_{3} T}\left|R_{1}\right|+\sqrt{\mu_{1}} T\left|R_{2}\right|+\sqrt{\mu_{3} T}\left|R_{3}\right|+\sqrt{\mu_{1}} T\left|R_{4}\right| .
$$

In view of (4.6), it is natural to split the tolerance into four terms: two of them related to the spatial discretization: $T O L_{M}, T O L_{m}$, and two of them related to the temporal discretization: $T O L_{K}, T O L_{k}$ and to require that

$$
\begin{gathered}
\max _{1 \leq q \leq Q} \frac{E_{q}^{\prime}}{M_{q}^{2}} \leq T O L_{M}, \max _{1 \leq q \leq Q} \frac{E_{q}^{\prime \prime}}{m_{q}^{2}} \leq T O L_{m}, \\
\max _{1 \leq q \leq Q} K_{q} E_{q}^{\prime \prime \prime} \leq T O L_{K}, \max _{1 \leq n \leq N} k_{n} E_{n} \leq T O L_{k} .
\end{gathered}
$$


For similar reasons to the ones above in Section 4.1, the splitting of $T O L$ will be such that:

$$
T O L_{M}+T O L_{m}=\frac{1}{10} T O L \text { and } T O L_{K}+T O L_{k}=\frac{9}{10} T O L
$$

Also, recall that we aim to compare the method with the one-level algorithm. In the non-adaptive case, we previously evidenced that the error of the two-level method with parameters $(M, m, K, k)$ is similar to the one of a one-level method with parameters $M$ and $k$, at least for some convenient values of $m$ and $K$ (see [12]); of course it can not be better than that error. Therefore we will ask for the two adaptive algorithms to yield close values of $k$ and $M$. In view of the different expressions of the estimators, this leads us to introduce a small parameter $\beta \ll 1$ and to set

$$
\begin{aligned}
& \text { TOL } L_{M}=\frac{1}{10} \beta T O L \text { and } T O L_{k}=\frac{9}{10} \beta T O L . \\
& T O L_{m}=\frac{1}{10}(1-\beta) T O L \text { and } T O L_{K}=\frac{9}{10}(1-\beta) T O L . \\
& \mathcal{T O} \mathcal{L}=\beta T O L
\end{aligned}
$$

This strategy is checked numerically in Section 5 below.

The algorithm proceeds as follows. At time $T_{q}$ (corresponding to a large time interval $J_{q}$ ), we compute the parameters $M_{q}, m_{q}, K_{q}$, the time-step $k_{n_{q-1}+1}$ corresponding to the first small time interval $I_{n} \subset J_{q}$ and $\left(V_{n_{q-1}+1}, W_{q}\right)$ by requiring that

$$
\begin{aligned}
\frac{E_{q}^{\prime}}{M_{q}^{2}} & \approx T O L_{M}, \frac{E_{q}^{\prime \prime}}{m_{q}^{2}} \approx T O L_{m}, \\
K_{q} E_{q}^{\prime \prime \prime} & \approx T O L_{K}, k_{n_{q-1}+1} E_{n_{q-1}+1} \approx T O L_{k}
\end{aligned}
$$

Next, we proceed with the successive small time intervals $I_{n} \subset J_{q}$. That is, for $n_{q-1}<n \leq n_{q}$, we compute successively the parameters $k_{n}$ and $V_{n}$ by requiring that:

$$
k_{n} E_{n} \approx T O L_{k} .
$$

Again the conditions (4.9), (4.10) are obtained thanks to some iterative procedure that converges in one or two iterations.

We conclude this section by some remarks concerning the numerical interpolation as well as the computation of the stability constants.

Practical implementation requires some numerical quadrature. We introduce an interpolation operator of order $l \geq m$ for equation (2.8a) and an interpolation operator of order of $L \geq M$ for equation (2.8b). It was shown in [12] that the use of two different operators is crucial for reducing the computing time and that a reasonable choice is $l=m+1$ and $L=M+1$. Numerical tests for the adaptive scheme show that this choice is indeed pertinent, see [14].

Concerning the stability constants, it is well known that the estimates of lemma 3.1 can not be used in practice due to their exponential dependence. Following [10], the constants are numerically estimated by solving (2.1), obtaining some error $e$ and solving the backward problem (3.17). The reader is refered to [10], [14] for more details. It is worth noting that the numerical values of the constants for the one-level and the two-level algorithms are quite similar, so that the constants can be evaluated trough the standard one-level method. 


\section{NUMERICAL EXPERIMENTS}

We present some numerical tests for one-dimensional problems.

Example 1.

We consider the following problem (see [17]):

$$
\left.\frac{\partial u}{\partial t}-(\sin x) \frac{\partial u}{\partial x}-\nu \frac{\partial^{2} u}{\partial x^{2}}=f \text { in }\right] 0,2 \pi[\times] 0, T[
$$

for which the characteristics curves are determined exactly. We choose $T=10, \nu=10^{-2}$ and consider the test function:

$$
u(x, t)=\sin (t) \sin \left(2 \arctan \left(10 \tan \left(\frac{x}{2}\right)\right)\right)+2 .
$$

The two adaptive algorithms were implemented but here we only present results concerning the two-level method. We refer to [14] for further considerations.

We first discuss the choice of $\beta$ in the decomposition (4.8) of the tolerance. For $T O L=0,5$, Table 1 presents the error $\mid\left(u-\left.(V+W)\right|_{T} /|u|_{T}\right.$ for various values of $\beta$.

TABLE 1. TOL $=0.5$
\begin{tabular}{|l|l|}
\hline$\beta$ & Error \\
\hline $1 / 5$ & $3.51 \times 10^{-3}$ \\
\hline $1 / 10$ & $1.76 \times 10^{-3}$ \\
\hline $1 / 20$ & $9.18 \times 10^{-4}$ \\
\hline $1 / 25$ & $7.59 \times 10^{-4}$ \\
\hline
\end{tabular}

TABLE 2. $T O L_{M}+T O L_{k}=0.05$.

\begin{tabular}{|l|l|l|}
\hline$\beta$ & Error & Running time \\
\hline $1 / 5$ & $1.79 \times 10^{-3}$ & $30.02 \mathrm{~s}$ \\
\hline $1 / 10$ & $1.76 \times 10^{-3}$ & $22.43 \mathrm{~s}$ \\
\hline $1 / 20$ & $1.79 \times 10^{-3}$ & $19.50 \mathrm{~s}$ \\
\hline $1 / 25$ & $1.9 \times 10^{-3}$ & $18.94 \mathrm{~s}$ \\
\hline
\end{tabular}

The error decreases with $\beta$. Indeed, $\beta$ being small, its value mainly influences the determination of $M$ and $k$. This leads to finer discretizations for smaller $\beta$.

Next, Table 2 provides the values of the error for various $\beta$, the quantity $T O L_{M}+T O L_{k}=\beta T O L$ being fixed (the value of $T O L$ varies). It is interesting to note that the error depends very slightly on $\beta$. Also, the smaller $\beta$ is, the quicker the algorithm runs. Indeed, then, $T O L_{m}$ increases, so that $m$ decreases leading to fewer operations (the corresponding FFTs are of order $m+1$ ). However note that $\beta$ should not be chosen too small since the error tends to increase for very small $\beta$ due to a less efficient choice of $m$ and $K$.

Next, Figure 1 shows the evolution of the parameters $M, m$ and $K, k$ with respect to time for $T O L=0,5$ and $\beta=1 / 10$. The variation of the parameters follows the time-periodicity of $u$. Also, it is worth noting that the ratio $K / k$ varies from 12 to 40 . This ratio is fixed and chosen a priori in [4] that deals with parabolic problems. Here the algorithm is able to determine all discretization parameters and their variation justifies the interest of adaptivity.

Now, we want to compare the performances of this algorithm with the ones of the one-level adaptive method. As already mentioned, we use the value $T O L$ (resp. $\mathcal{T} \mathcal{O} \mathcal{L}=\beta T O L$ ) for the tolerance of the two-level (resp. one-level) method. Table 3 gives the error and the computing time for the two methods with different values of TOL and $\beta=1 / 10$.

We see that the errors for the two methods are similar. Of course this justifies our choice of the respective tolerances. The errors being similar, the computing times of the two methods can be compared. The two-level procedure yields a gain of the order of $50 \%$. 

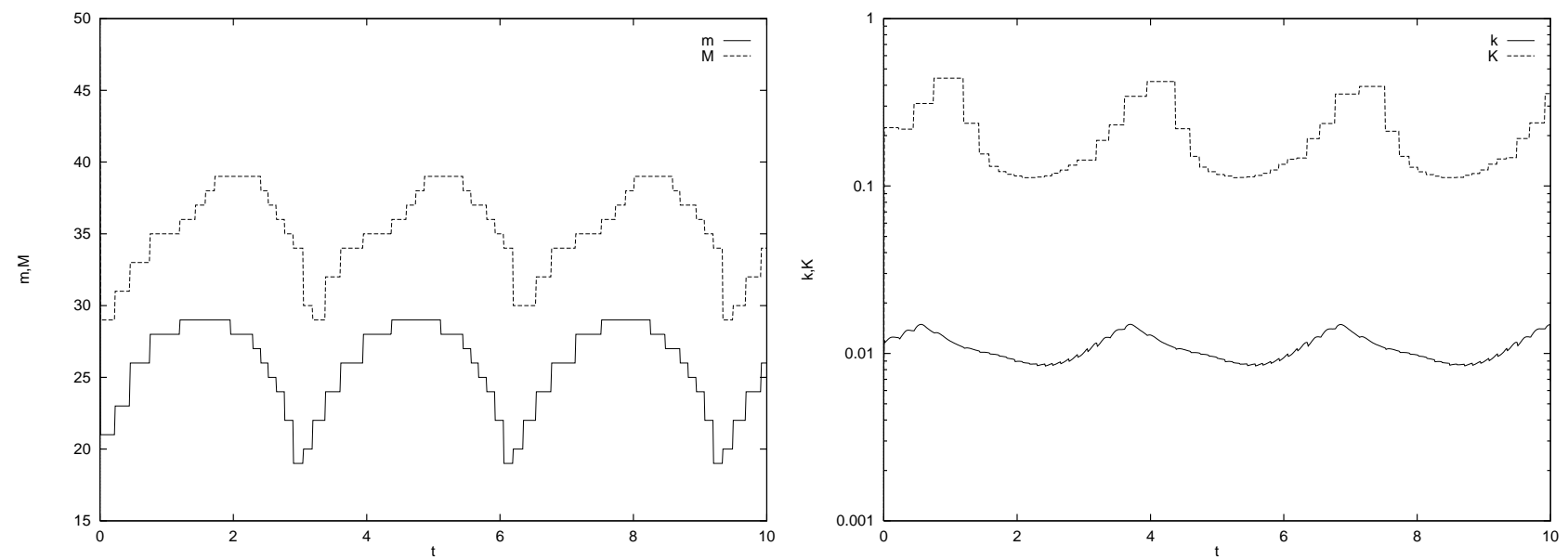

Figure 1. (a) $m_{q}, M_{q}$ in function of $t$; (b) $k_{n}, K_{q}$ in function of $t$.

TABLE 3. Error and computing time for the two methods.

\begin{tabular}{|l|l|l|l|l|l|}
\hline TOL & Error(1 level) & Error(2 levels) & Comp. time (1 level) & Comp. time $(2$ levels $)$ & Ratio \\
\hline 1 & $3.5 \times 10^{-3}$ & $3.5 \times 10^{-3}$ & $19.19 \mathrm{~s}$ & $9.77 \mathrm{~s}$ & 0.51 \\
\hline $5 \times 10^{-1}$ & $1.8 \times 10^{-3}$ & $1.8 \times 10^{-3}$ & $41 \mathrm{~s}$ & $22.5 \mathrm{~s}$ & 0.55 \\
\hline $10^{-1}$ & $3.9 \times 10^{-4}$ & $3.9 \times 10^{-4}$ & $235.65 \mathrm{~s}$ & $142.16 \mathrm{~s}$ & 0.6 \\
\hline
\end{tabular}

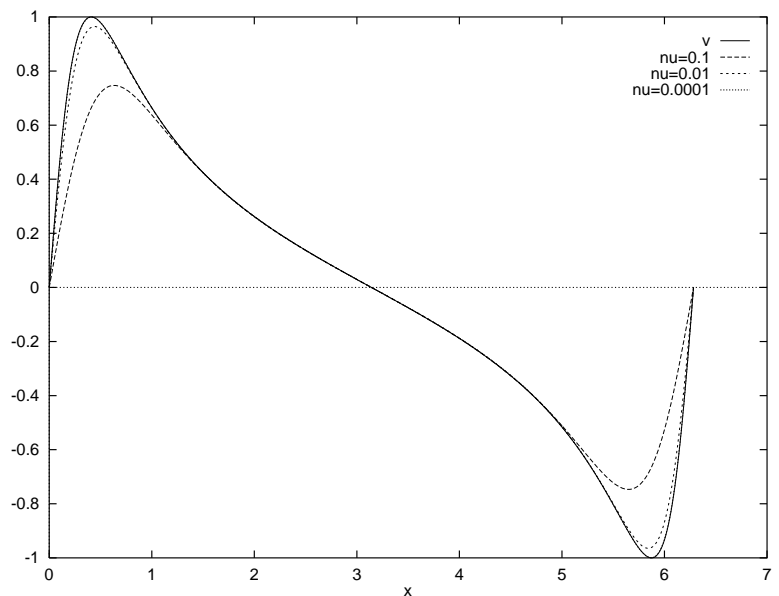

Figure 2. Convergence of the two-level method.

Example 2.

We now want to test the behavior of the algorithm for small values of $\nu$. Following [17], we consider the equation (5.1) with $f=0$ and the initial condition $u_{0}(x)=\sin x$. Then, the solution of the hyperbolic problem $(\nu=0)$ is:

$$
v(x, t)=\sin \left(2 \arctan \left(e^{t} \tan \left(\frac{x}{2}\right)\right)\right)
$$

Figure 2 presents $v$ at $t=T_{\max }=\pi / 2$ as a function of $x \in[0,2 \pi]$ and the approximate solution obtained thanks 
to the two-level method for various values of $\nu\left(\nu=10^{-1}, 10^{-2}, 10^{-4}\right)$. Clearly, the multi-level approximations converge to $v$ as $\nu \rightarrow 0$. This indicates the stability of the algorithm for $\nu \ll 1$.

The influence of $\beta$ can be studied as in Example 1. The conclusions are completely similar and the details are omitted.

Let us now investigate the performances of the algorithm. We choose $\nu=10^{-10}$ and compare the one-level and the two-level adaptive methods. For a similar error, Table 4 gives the computing times of the two methods and their ratio.

TABLE 4. Running times of the two methods.

\begin{tabular}{|l|l|l|l|}
\hline Error & Comp. time (1 level) & Comp. time (2 levels) & Ratio \\
\hline $3.3 \times 10^{-3}$ & $6.93 \mathrm{~s}$ & $2.89 \mathrm{~s}$ & 0.41 \\
\hline $1.6 \times 10^{-3}$ & $16.51 \mathrm{~s}$ & $7.61 \mathrm{~s}$ & 0.46 \\
\hline $8.0 \times 10^{-4}$ & $47.94 \mathrm{~s}$ & $17.37 \mathrm{~s}$ & 0.36 \\
\hline $3.2 \times 10^{-4}$ & $115.42 \mathrm{~s}$ & $47.71 \mathrm{~s}$ & 0.41 \\
\hline $1.6 \times 10^{-4}$ & $270.08 \mathrm{~s}$ & $106.23 \mathrm{~s}$ & 0.39 \\
\hline
\end{tabular}

Now the gain in computing time due to the multi-level procedure is of the order of $65 \%$. It is worth noting that it is much more important than the one in [12] for the non-adaptive algorithm (that was of order of $25 \%$ ).

\section{REFERENCES}

[1] M. Bercovier, O. Pironneau and V. Sastri, Finite elements and characteristics for some parabolic-hyperbolic problems. Appl. Math. Modelling 7 (1983) 89-96.

[2] K. Boukir, Y. Maday, B. Metivet and R. Razafindrakoto, A high-order characteristics/finite element method for imcompressible Navier-Stokes equations, Rapport de l'Université Pierre et Marie Curie, R 92032 (1992).

[3] J.B. Burie and M. Marion, Multi-level methods in space and time for Navier-Stokes equations. SIAM J. Numer. Anal. 34 (1997) 1574-1599.

[4] J.B. Burie and M. Marion, Adaptative multi-level methods in space and time for paraboloc problems- The periodic case. Math. of Comp. (to appear).

[5] A. Debussche, T. Dubois and R. Temam, The nonlinear Galerkin method: A multi-scale method applied to the simulation of turbulent flows. Theoret. Comput. Fluid Dynamics 7 (1995) 279-315.

[6] J. Douglas and T.F. Russel, Numerical methods for convection dominated diffusion problems based on combining the method of caracteristics with finite element methods or finite difference method. SIAM J. Numer. Anal. 19 (1982) 871-885.

[7] T. Dubois, Simulation numérique d'écoulement homogènes et non-homogènes par des méthodes multi-résolution, Thèse, Université Paris-Sud (1993).

[8] K. Eriksson and C. Johnson, Adaptative finite element methods for parabolic problems I: A linear model problem. SIAM J. Numer. Anal. 28 (1991) 43-77.

[9] C. Foias, O. Manley and R. Temam, Modelling of the interaction of small and large eddies in two-dimensional turbulent flows. $M^{2} A N 22$ (1998) 93-114.

[10] P. Houston and E. Suli, Adaptative Lagrange-Galerkin methods for unsteady convection-dominated diffusion problems, Oxford University Computing Laboratory Report, 95/24 (1995).

[11] F. Jauberteau, Résolution numérique des équations de Navier-Stokes instationnaires par méthodes spectrales. Méthode de Galerkin non linéaire, Thèse, Université Paris-Sud (1990).

[12] M. Marion and A. Mollard, A multi-level characteristics method for periodic convection-dominated diffusion problems. Numer. Meth. PDEs. (to appear).

[13] M. Marion and J. Xu, Error estimates on a new nonlinear Galerkin method based on two-grid finite elements. SIAM J. Numer. Anal. 32 (1995) 1170-1184.

[14] A. Mollard, Méthodes de caractéristiques multi-niveaux en espace et en temps pour une équation de convection-diffusion - Cas d'une approximation pseudo-spectrale, Thèse, École Centrale de Lyon (1998).

[15] O. Pironneau, Finite element methods for fluids, Masson (1989).

[16] E. Suli, Convergence and nonlinear stability of the Lagrange-Galerkin method for the Navier-Stokes Equations. Numer. Math. 53 (1988) 459-483.

[17] E. Suli and A.F. Ware, A spectral method of characteristics for hyperbolic problems. SIAM. J. Numer. Anal. 28 (1991) 423-445. 